\title{
Sonographic Venous Velocity Index Identifies Patients with Chronic Kidney Disease and Severe Diastolic Dysfunction
}

\section{(ㄷ) (i) (우) $\Theta$}

\author{
Authors \\ Markus Meier $^{1 *}$, Wolfram Johannes Jabs ${ }^{2 *}$, Maria Guthmann ${ }^{3}$, Gesa Geppert ${ }^{1}$, Ali Aydin ${ }^{3}$, Martin Nitschke
}

Affiliations

1 Nephrology Center Reinbek and Geesthacht, Outpatient Clinic, Reinbek, Germany

2 Vivantes Klinikum im Friedrichshain, Nephrology, Berlin, Germany

3 Krankenhaus-Reinbek Sankt Adolf-Stift, Cardiology, Reinbek, Germany

4 Universitat zu Lubeck Sektion Medizin, Medical Clinic I, Lubeck, Germany

Key words

cardiorenal syndrome, renal ultrasound, renal venous

congestion, chronic kidney disease

received 03.02 .2018

revised $\quad 04.06 .2018$

accepted 22.07.2018

Bibliography

DOI https://doi.org/10.1055/a-0684-9483

Ultrasound Int Open 2018; 4: E142-E148

(c) Georg Thieme Verlag KG Stuttgart · New York

ISSN 2199-7152

Correspondence

Dr. Markus Meier, MD, PhD

Nephrology Center Reinbek and Geesthacht

Outpatient Clinic

Am Ladenzentrum 8

21465 Reinbek

Germany

Tel.: +49/40/7277 860, Fax: + 49/40/72778615

markus.meier@dialyse-reinbek.de

\section{ABSTRACT}

Objective Diagnosing cardiorenal syndrome (CRS) in patients with chronic kidney disease (CKD) continues to remain challenging in outpatient practice. In this study, we investigate whether a newly developed venous velocity ultrasound index (VVI) can differentiate between patients with CRS and patients with CKD of other cause or normal renal function (NRF).

Methods Patients with CRS $(n=30)$, CKD $(n=30)$, and NRF $(n=30)$ were included in the study. For each patient, duplex ultrasound scans of intrarenal segmental veins were retrospectively analyzed. The VVI was calculated from the renal venous doppler curve as the ratio of the maximal positive venous velocity to the maximal negative venous velocity. Patients with CRS were compared to age-matched controls with NRF and to GFR-matched controls with CKD.

Results: The GFRs of patients with CRS and those with CKD were comparable ( $26.4 \pm 5$ and $25.6 \pm 7 \mathrm{ml} / \mathrm{min} / \mathrm{m} 2)$, as was the age in patients with CRS and NRF $(6 \pm 12$ years and $68 \pm 16$ years, respectively). There was no significant difference in ejection fraction between patients with CRS and those with CKD ( $44.2 \pm 6.2 \%$ vs. $47.4 \pm 7.2$ ), but there was a significant decrease compared to those with $\operatorname{NRF}(52.6 \pm 5.1, \mathrm{p}<0.01)$. The VVI was significantly higher in the CRS group $(0.81 \pm 0.18)$ compared to the CKD group $(0.18 \pm 0.17, p<0.01)$ or NRF group $(0.22 \pm 0.20, p<0.01)$. The positive predictability of CRS was $96.4 \%$ in patients with VVI values of $>0.6$.

Conclusion The newly developed VVI was useful in successfully predicting severe diastolic dysfunction (CRS) in patients with severe kidney injury in outpatient care.

\section{Introduction}

The association between cardiovascular diseases and chronic kidney disease (CKD) was first described by Ronco et al. in 2008 [1]. They defined cardiorenal syndrome (CRS) as a disorder characterized by the acute or chronic dysfunction of either of these systems as a result of a dysfunction of the other [1]. Recent reviews have

*both authors contributed equally to the work. shown that despite the recognition of this association between the two conditions, there still remains a lack of clarity regarding the pathogenesis and management of CRS [2,3].

CRS has been classified into five types, and type 4 CRS marks a state of CKD that compromises cardiac function, leads to cardiac hypertrophy, and increases the risk for cardiovascular events [1].

Although CRS has been studied extensively, there are no gold standard criteria for the diagnosis of CRS, since CRS describes only 
an interdependence between kidney and cardiac dysfunction. Therefore, the diagnosis of CRS is usually established if the estimated glomerular filtration rate (eGFR) declines in the clinical course of patients without an underlying renal disease but simultaneous worsening of cardiac function detected by echocardiography. (1-3). However, this distinction may be challenging in patients with CKD and additional suspected CRS (3).

The pathogenic changes associated with stage four CKD are characterized by post-translation modification of proteins, hyperphosphatemia, as well as vascular abnormalities such as atherosclerosis and arteriosclerosis [4]. End-stage renal disease causes a state of chronic inflammation, which leads to a phenomenon known as malnutrition-inflammation-atherosclerosis syndrome [5]. Considering this pathological background, renal congestion resulting from changes in the renal vasculature can be considered a characteristic feature in patients with CRS.

On the other hand, congestive heart failure (CHF) is characterized by volume overload and increased central venous pressure [6]. Thus, patients with severe HF typically present with hepatic and renal congestion leading to deterioration of renal blood flow that contributes to the development of CRS [7].

These alterations can be studied by Doppler ultrasonography and may be reflected as changes in the ultrasound spectrum of intrarenal veins. Therefore, we thought that it may be possible to detect CRS early in patients on the basis of the Doppler ultrasonography findings of their renal veins.

With this in mind, we formulated a new parameter, the venous velocity ultrasound index ( $\mathrm{VVI}$ ) based on the velocity of blood flow in renal veins. In this study, we aimed to investigate whether this new index can help differentiate between patients with CRS and those with CKD or normal renal function (NRF).

\section{Patients and Methods}

We conducted a retrospective analysis of 90 patients treated at a nephrology outpatient center in Germany. The first group of patients were those with CRS ( $n=30$; CRS group), who were assigned to a nephrologist by general physicians or cardiologists for the diagnosis and co-treatment of CKD, which implied that these patients had concomitant cardiovascular and renal dysfunction. Diagnosis of CRS in these patients was made according to Ronco et al. 2017 [8]. Briefly, only patients with chronic kidney disease at stage 4 (eGFR 15 to $30 \mathrm{ml} / \mathrm{min} / 1.73 \mathrm{~m}^{2}$ ) without proteinuria and concurrent severe diastolic dysfunction (grade II or III) and normal (HFpEF) or mid-range reduced (HFmrEF) ejection fraction were included in the CRS group. Kidney function of all patients in the CRS group worsened within the last 12 months, which could not be explained by inherited or acquired chronic renal disease. Additionally, a second group comprised of 30 patients with chronic kidney disease (CKD) secondary to causes other than CRS was enrolled. This group served as the matched control group (CKD group) in terms of the glomerular filtration rate (GFR). Another group of the same size $(n=30)$ was also included in this study to serve as the age-matched control group. This group consisted of living kidney donors who had normal function of the remnant kidney and cardiovascular system (NRF group). These subjects had donated their kidneys be- tween 2007 and 2014 at the transplant center at the University of Lubeck.

Patients in the CRS and CKD groups had undergone kidney ultrasound examination performed in the nephrology outpatient center by three experienced investigators and echocardiographic examination at the cardiology department of the Hospital of Reinbek, St. Adolf Stift between 2014 and 2017. The NRF group had undergone both ultrasound and echocardiographic examination at the Medical Department of the University of Lubeck between 2007 and 2014, as described previously $[9,10]$. The renal ultrasound examinations of these patients had been performed by the same three experienced investigators (MM; MN, WJ) who performed the investigations of the CKD and CRS group in the same standardized protocol (described below) a few years later. Thus, we were able to minimize intra- and inter-observer variability.

Data for the CRS and CKD groups were collected from the medical records maintained at our center, while the data for the NRF group were obtained from the prospectively maintained registry of patients at the transplant Center of Lübeck and the Nephrology outpatient Center of Reinbek.

This study was conducted in keeping with the Helsinki Declaration of 1975 and the study protocol was approved by the local ethics committee at our university.

\section{Kidney ultrasound examination}

Data regarding both routine grayscale and duplex ultrasound scans were obtained for all enrolled patients. The standard ultrasound examination protocol applied for the patients included in the current study has been outlined previously $[9,10]$ and is as follows: UItrasound examination was performed in the supine position during the morning hours by one of the three authors using the same transducers (multifrequency probes, curved array, C4-1, C9-3 or C6-2, Zonare Medical Systems, Mountain View, Ca, USA) and devices (Z.one and ZS3, Zonare Medical Systems, Mountain View, CA, USA). The following parameters were measured and recorded during each ultrasound scan: the cortex-pelvis proportion (CPP), resistive index (RI), pulsatility index (PI), and venous velocity (VV). The definitions for these parameters have been described previously [10]. This protocol was established at the transplant center in 2006 and adopted by our outpatient clinic five years later.

The velocity of the renal veins was measured for at least three different renal segments. The average of the three sets of measurements was used for further analysis. As a cautionary measure, the patients were asked to hold their breath in deep inspiration during the determination of VVI since breathing could influence the VVI measurement. The VVI was measured using the maximal positive and maximal negative venous velocity determined from the biphasic pulse-wave Doppler flow curve on the basis of the following formula incorporated into the built-in ultrasound software.

\section{Venous velocity index $(\mathrm{VVI})=$ maximal positive velocity/maximal negative velocity}

In cases where the segmental venous flow was found to be negative and monophasic, the maximal positive velocity was considered to be " 0 ," which meant that the VVI would also be " 0 ."

In the CKD and CRS group, all indices were measured for both kidneys and the average of the two values was taken as the meas- 
urement for a given patient. This did not apply to the NRF patients, since they had only one remnant kidney.

\section{Echocardiographic examination}

All patients underwent transthoracic echocardiography, performed in accordance with the national and international standards. The echocardiography scans were performed by experienced technicians after allowing a resting period of 20 minutes for stabilization [11]. The devices used in all patients were either Vivid 7 or Vivid 9 (GE Healthcare, Frankfurt, Germany), and all measured parameters were determined offline using the EchoPac software (GE Healthcare, Frankfurt, Germany).

Left ventricular (LV) systolic function was determined from the LV ejection fraction using the modified Simpson's rule, with biplane LV volume analysis [12]. LV diastolic function was determined on the basis of the values of maximal early-diastolic velocity $\left(E_{\max }\right)$ and maximal late-diastolic $\left(A_{\max }\right)$ velocity of the mitral valve inflow obtained from pulsed-wave Doppler measurement made in the apical long-axis view. The measurements were performed at a distance of 1 to $2 \mathrm{~cm}$ superior to the mitral ring, at the junction of the mitral leaflets to the chord strands; improper Doppler angles for measurement were avoided. Peak mitral $E$ and $A$ wave velocities were measured to calculate the E/A-ratio. Right ventricular (RV) function was then assessed on the basis of tricuspid annular plane systolic excursion (TAPSE) determined in the M-mode. Furthermore, RV-focused images in the apical four-chamber view were also acquired for further analysis.

- Table 1 Patient characteristics.

\section{Results}

\section{Baseline parameters}

The baseline characteristics of the patients in the three groups are provided in $>$ Table 1. The groups CRS and CKD were similar in terms of the estimated GFR $\left(26.4 \pm 5\right.$ and $25.6 \pm 7 \mathrm{ml} / \mathrm{min} / \mathrm{m}^{2}$, respectively). On the other hand, the CRS group was comparable to the NFR group in terms of age ( $68 \pm 12$ years and $68 \pm 16$ years, respectively). With respect to the other parameters, the frequency of CKD-associated comorbidities such as hypertension, hypertensive nephropathy, and diabetic nephropathy was the highest in the CKD, as expected ( $\vee$ Table $\mathbf{1}$ ).

\section{Echocardiography measurements}

With respect to the echocardiography parameters, the systolic ejection fractions in the CRS and CKD groups were similar in the two remaining groups, but significantly lower than in the NRF group $(\mathrm{p}<0.01$, ANOVA on ranks) ( $\vee$ Table 2$)$.

The most important features noted in CRS patients were tricuspid insufficiency of a grade greater than or equal to II and vena cava dilatation (of more than $20 \mathrm{~mm}$ ), which were observed in all and almost all (80\%) of the patients in this group. Furthermore, grade III diastolic ventricular dysfunction was noted only (60\%) and most often ( $40 \%$ ) in the CRS patients ( $\triangleright$ Table 2 ).

\section{Ultrasound examination}

The RI and PI showed slightly higher values in the CKD group compared to the NRF group, but the difference between these values

\begin{tabular}{|c|c|c|c|}
\hline Criterion & CRS $(n=30)$ & NRF (age-matched) $(n=30)$ & CKD (GFR-matched) $(n=30)$ \\
\hline Age (years) & $68 \pm 12$ & $68 \pm 16$ & $62 \pm 28$ \\
\hline $\operatorname{Sex}(w / m)$ & $12 / 18$ & $16 / 14$ & $17 / 13$ \\
\hline BMI $\left(\mathrm{kg} / \mathrm{m}^{2}\right)$ & $23.3 \pm 4.2$ & $25.2 \pm 3.6$ & $26.5 \pm 4.8$ \\
\hline eGFR (CKD-Epi) (ml/min/1.73 m²) & $26.4 \pm 5$ & $81.1 \pm 9^{*}$ & $25.6 \pm 7$ \\
\hline Albuminuria (mg/g creatinine) & $68 \pm 134$ & $15 \pm 11^{*}$ & $156 \pm 231$ \\
\hline \multicolumn{4}{|l|}{ Blood pressure $(\mathrm{mmHg})$} \\
\hline Systolic & $104 \pm 14$ & $118 \pm 14$ & $122 \pm 16$ \\
\hline Diastolic & $72 \pm 12$ & $78 \pm 12$ & $76 \pm 12$ \\
\hline Heart rate (bpm) & $67 \pm 9$ & $74 \pm 11$ & $68 \pm 8$ \\
\hline History of hypertension & 21 & 8 & 22 \\
\hline Hypertensive nephropathy & 2 & 0 & 11 \\
\hline Diabetes mellitus & 12 & 2 & 8 \\
\hline Diabetic nephropathy & 0 & 0 & 8 \\
\hline ADPKD & 0 & 0 & 5 \\
\hline Glomerulonephritis & 0 & 0 & 4 \\
\hline Interstitial nephritis & 0 & 0 & 1 \\
\hline Cyclosporine toxicity & 0 & 0 & 1 \\
\hline Cardiorenal syndrome type 2 & $30(100 \%)$ & $0(0 \%)$ & $0(0 \%)$ \\
\hline
\end{tabular}


> Table 2 Echocardiographic parameters.

\begin{tabular}{|c|c|c|c|}
\hline Parameter & CRS $n=30$ & NRF (age-matched) $n=30$ & CKD (GFR-matched) $n=30$ \\
\hline \multicolumn{4}{|l|}{ Systolic left ventricular function } \\
\hline (Ejection fraction, Teichholz) \% & $44.2 \pm 6.2$ & $52.6 \pm 5.1^{*}$ & $47.4 \pm 7.2$ \\
\hline \multicolumn{4}{|l|}{ Diastolic ventricular dysfunction } \\
\hline None & $0(0 \%)^{\#}$ & $22(73.3 \%)$ & $20(66.6 \%)$ \\
\hline Grade I & $0(0 \%)^{\#}$ & $8(26.6 \%)$ & $18(60 \%)$ \\
\hline Grade II & $12(40 \%)^{\#}$ & $0(0 \%)$ & $2(6 \%)$ \\
\hline Grade III & $18(60 \%)^{\#}$ & $0(0 \%)$ & $0(0 \%)$ \\
\hline Tricuspid insufficiency $\geq 1^{\circ}$ & $30(100 \%)^{\#}$ & $0(0 \%)$ & $3(10 \%)$ \\
\hline TAPSE & $16.4 \pm 1.8^{\#}$ & $24.9 \pm 1.9$ & $23.5 \pm 1.8$ \\
\hline RVEDV (mm) & $31.8 \pm 2.8$ & $30.2 \pm 3.2$ & $30.8 \pm 2.7$ \\
\hline Aortic valve stenosis $\geq 11^{\circ}$ & $0(0 \%)$ & $0(0 \%)$ & $2(6.6 \%)$ \\
\hline Mitral valve insufficiency $\geq \|^{\circ}$ & $12(40 \%)$ & $0(0 \%)$ & $8(26.6 \%)$ \\
\hline Pericardial effusion & $0(0 \%)$ & $0(0 \%)$ & $1(3.3 \%)$ \\
\hline Vena cava dilated (> $20 \mathrm{~mm}$ ) & $24(80 \%)^{\#}$ & $0(0 \%)$ & $6(20 \%)$ \\
\hline Pleural effusion & $5(16.6 \%)$ & $0(0 \%)$ & $2(6.6 \%)$ \\
\hline
\end{tabular}

TAPSE: tricuspid annular plane systolic excursion, RVEDV: right ventricular end diastolic volume; * parameters with p-value $<0.05$ compared to CRS (ANOVA on ranks); \#parameter with p-value $<0.05$ compared to NRF and CKD (Fisher's exact test)

$>$ Table 3 Renal ultrasound parameters.

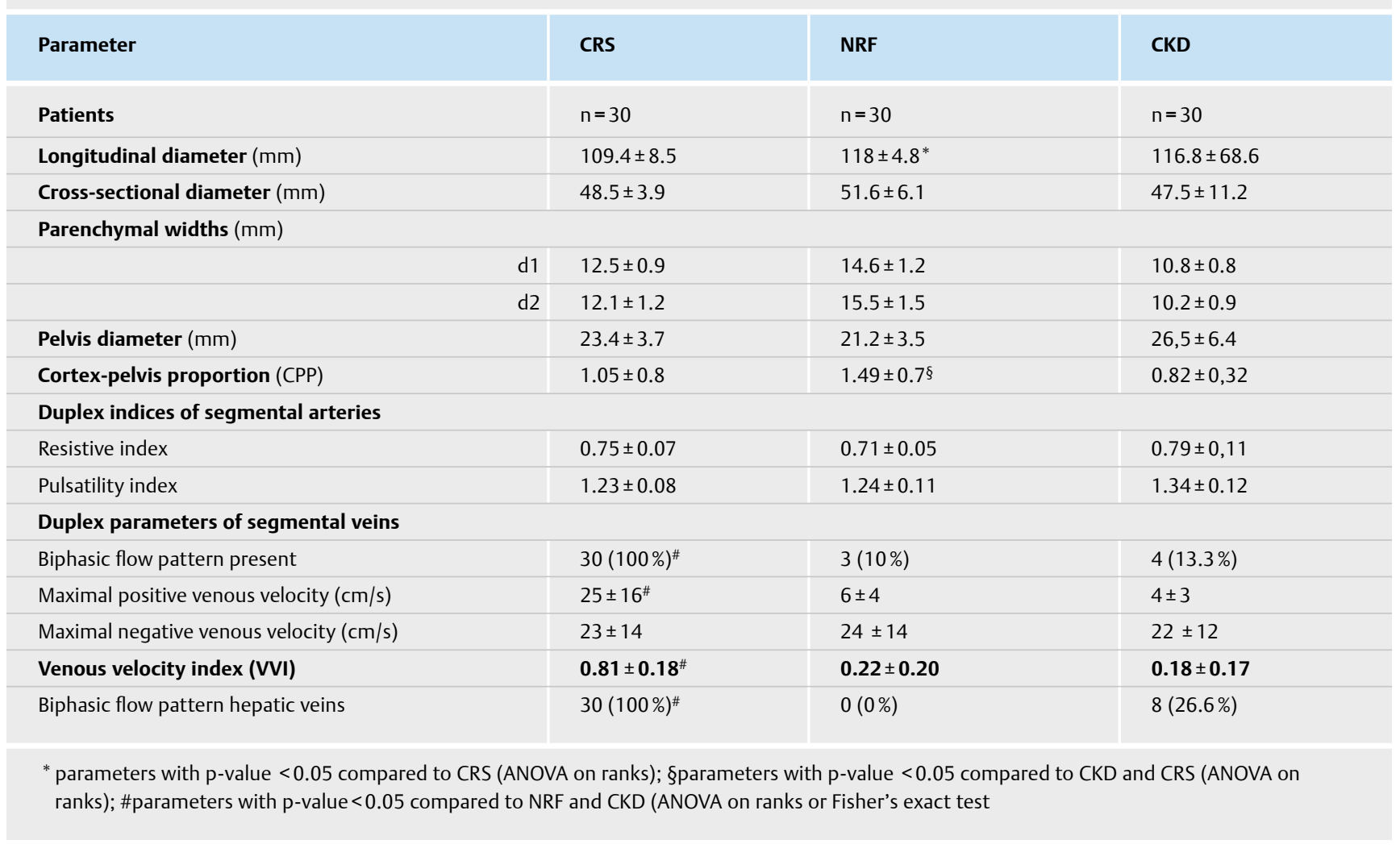


when comparing the CRS and CKD patients was only minimal ( $\triangleright$ Table 3). On the other hand, the VVI was significantly higher in the CRS group $(0.81 \pm 0.18)$ than in the CKD group $(0.18 \pm 0.17$, $\mathrm{p}<0.01$, ANOVA on ranks) and the NRF group $(0.22 \pm 0.20, \mathrm{p}<0.01$, ANOVA on ranks) ( $\triangleright$ Table 3$)$. Thus, the new index was much more

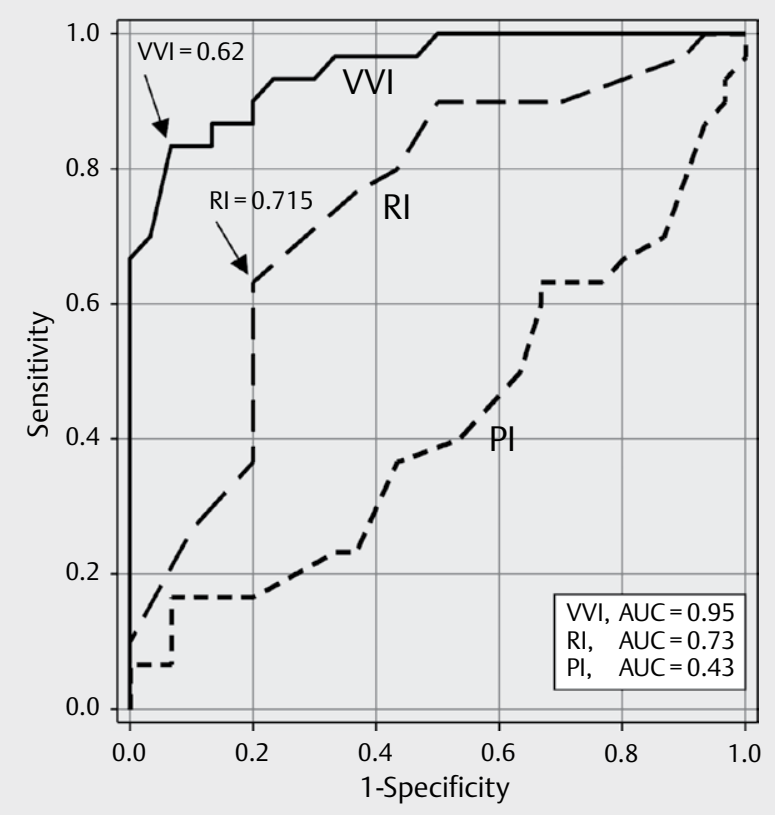

- Fig. 1 Diagnosis of cardiorenal syndrome with the duplex ultrasound parameters venous velocity index (VVI), resistive index (RI) and pulsatility index (PI). The area under the receiver-operating characteristic curve (AUC) to predict diagnosis of CRS was 0.95 for VVI, 0.73 for $\mathrm{RI}$ and 0.43 for PI. VVI values of 0.61 (arrow) and RI values of 0.715 (arrow) had the highest accuracy. The PI has a lower probability to detect CRS as randomly selected controls. effective in predicting CRS. The area under the receiver-operating characteristic curve (ROC) to predict diagnosis of CRS was 0.95 for VVI, 0.73 for RI and 0.43 for PI ( $\triangleright$ Fig. 1). In fact, the positive predictability for CRS using VVI values of $>0.6$ was high, at $96.4 \%$ in patients with VVI. Moreover, correlation analysis shows significant association between the VVI and the degree of diastolic dysfunction ( $p<0.001, r=0.93$, Spearman's rank-order correlation). In contrast, the standard duplex ultrasound parameters RI and PI did not correlate with the severity of diastolic dysfunction $(p=0.26, r=0.38$ and $p=0,32, r=0,29$, respectively, Spearman's rank-order correlation).

The biphasic flow pattern in the renal segmental veins was noted in all patients with CRS, while the frequency of this flow pattern in the remaining two groups was similar and only about one-tenth ( Fig. 2-4). Furthermore, CRS patients showed the biphasic flow pattern in the case of the hepatic veins.

\section{Discussion}

Although CRS has been defined and classified into different types, these developments have been fairly recent, occurring only within the last decade [13]. There still remains a significant amount of ambiguity among clinicians regarding the pathogenesis and best treatment options for this complex syndrome [2,3]. Renal dysfunction has been known to compromise cardiac function. In fact, even a small elevation in urinary albumin has been known to cause a twoto four-fold increase in the risk of cardiovascular disease [3]. However, the condition remains difficult to diagnose, which makes it difficult to ensure timely intervention.

Use of Doppler ultrasound parameters in the detection of the cardiorenal syndrome $[14,15]$ has been advocated by several investigators. Doppler ultrasound is particularly suitable for patients with renal dysfunction because it is noninvasive and does not carry the risk of renal complications, unlike other investigative modali-
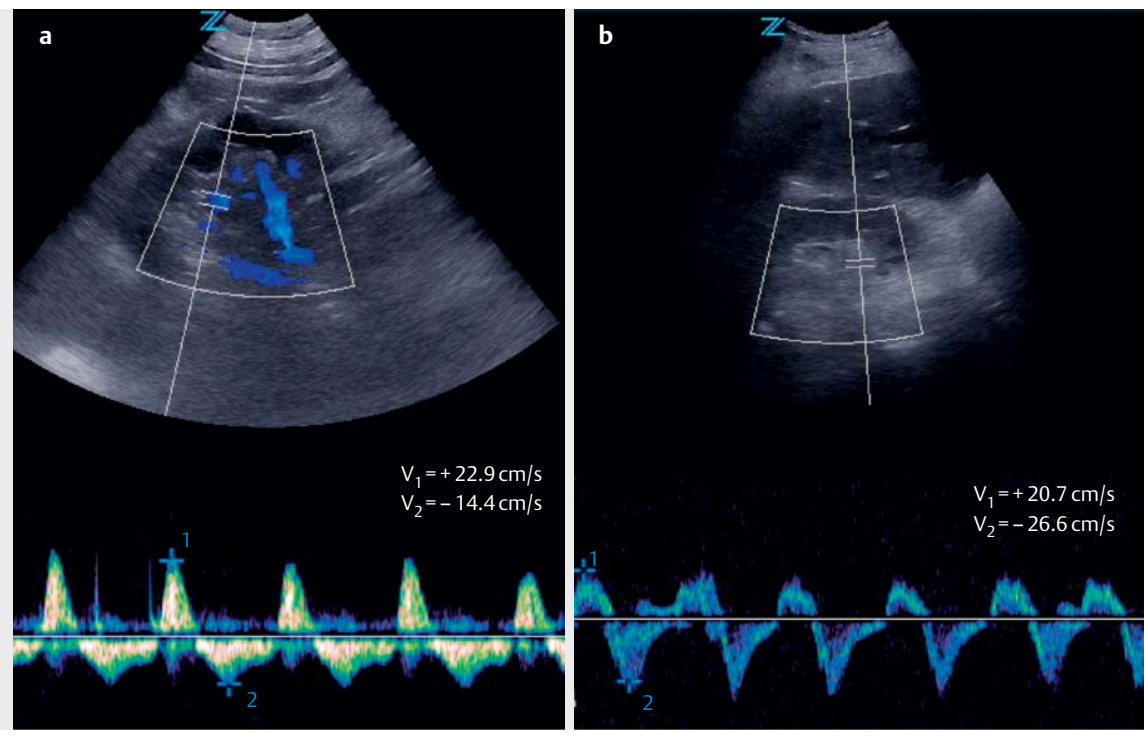

- Fig. 2 Exemplary kidney duplex ultrasound investigations of patients with cardiorenal syndrome (CRS) and grade III (a) and grade II (b) diastolic dysfunction. Venous Doppler spectrum of both patients demonstrates a biphasic flow pattern. Calculation of the venous duplex index (VVI) in patient $\mathrm{A}: \mathrm{VVI}=\mathrm{V} 1 / \mathrm{V} 2=1.6$; patient $\mathrm{B}: \mathrm{VVI}=\mathrm{V} 1 / \mathrm{V} 2=0.77$ 

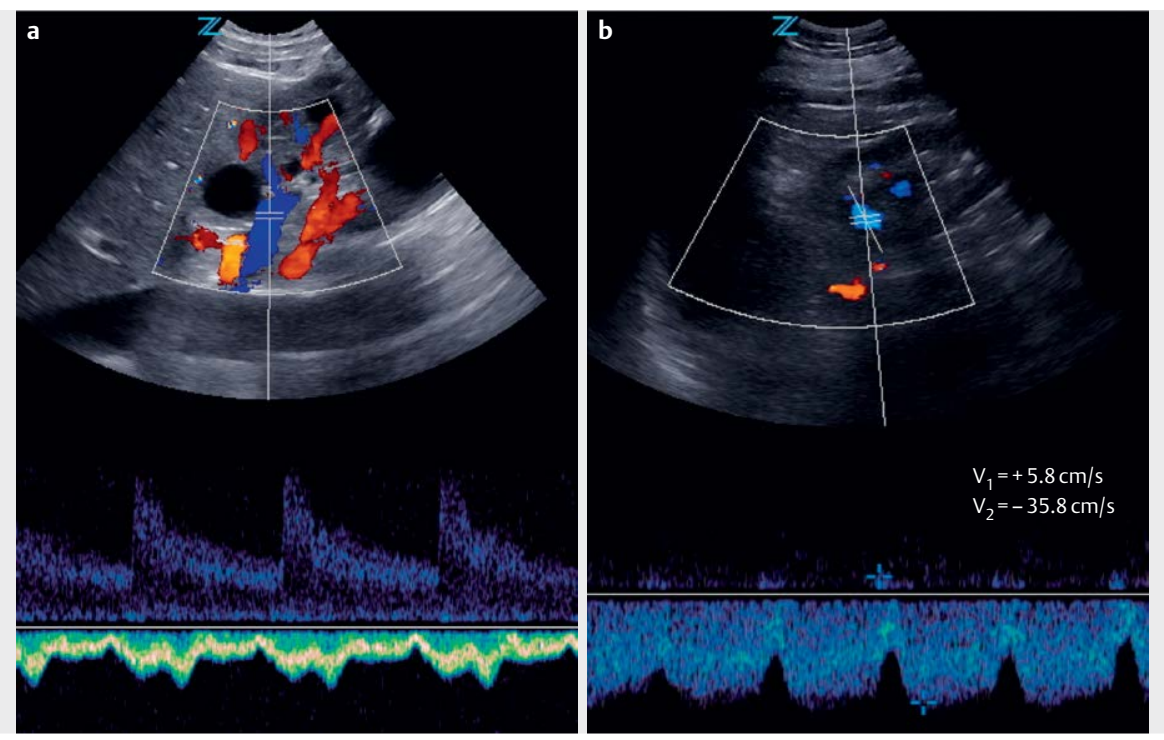

- Fig. 3 Exemplary kidney duplex ultrasound investigations of a patient with CKD. Venous Doppler spectrum of a patient with ADPKD (a) shows a monophasic negative flow pattern, whereas a patient with nephrosclerosis shows a biphasic flow pattern (b). Calculation of the venous duplex index $(\mathrm{VVI})$ in patient $\mathrm{A}$ (no positive flow): $\mathrm{VVI}=0$; patient $\mathrm{B}: \mathrm{VVI}=\mathrm{V} 1 / \mathrm{V} 2=0.16$.
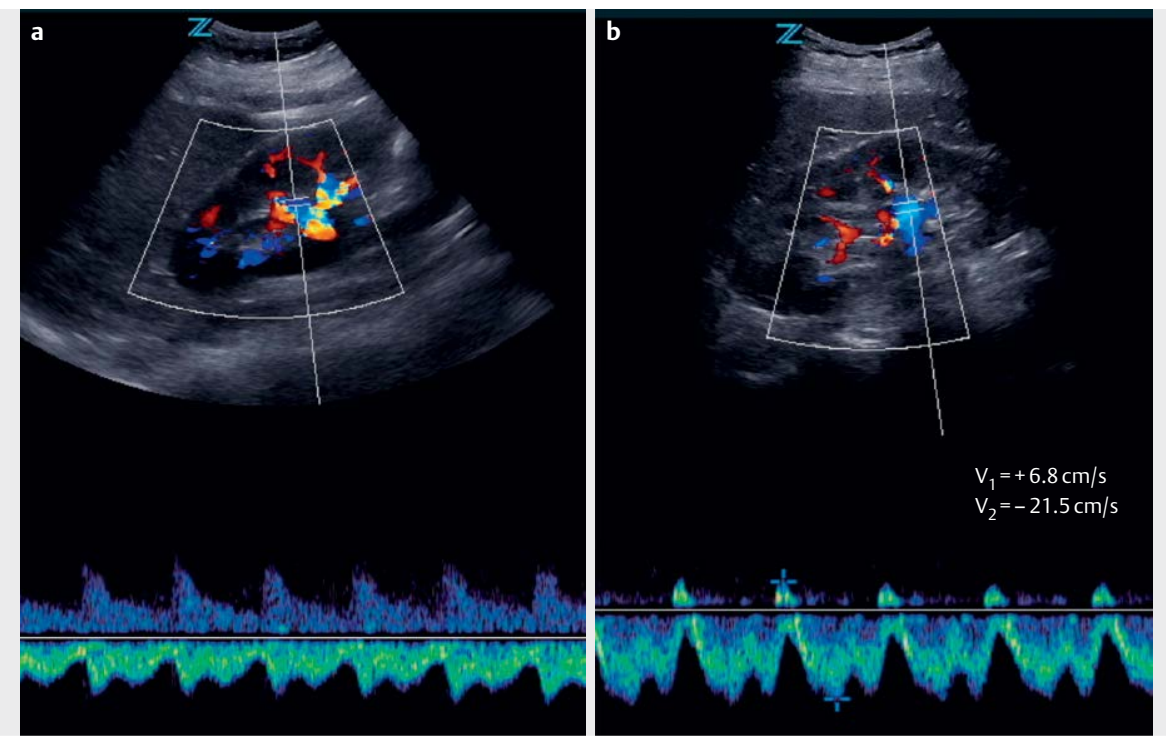

- Fig. 4 Exemplary kidney ultrasound investigations of living kidney donors with normal remnant kidney and cardiac function. Venous Doppler spectrum of a 65-year-old patient (a) shows a monophasic negative flow pattern, whereas a 58-year-old patient (b) shows a biphasic flow pattern (b). Calculation of the venous duplex index (VVI) in patient $\mathrm{A}$ (no positive flow): $\mathrm{VVI}=0$; patient $\mathrm{B}: \mathrm{VVI}=\mathrm{V} 1 / \mathrm{V} 2=0.32$.

ties such as contrast-enhanced computed tomography and digital subtraction angiography.

Among the various Doppler ultrasound parameters, RI has been gaining ground as a useful factor in the prediction of cardiorenal syndrome $[14,15]$. A renal $R I$ of $\geq 0.70$ is generally considered indicative of abnormal renal function and poor prognosis $[16,17]$. However, contrary to this, we found these levels of RI in our patients with NRF. This finding may be attributed to the fact that our patients with NRF had only a single kidney since they were living donors. However, it is unclear whether and how this factor would contribute to this discrepancy in our study and previous ones.
Furthermore, we found that the VVI had greater value in predicting CRS compared to the RI and PI. This finding is consistent with those reported by lida et al. [18] and Nijst et al. [19]. They analyzed the intrarenal venous flow patterns in patients with heart failure $[18,19]$ and found that, compared to the RI, the venous flow patterns were independent predictors of a compromised heart [18] and correlate with intravascular volume expansion [19]. They attributed this to the close association between the renal venous flow patterns and the renal congestion associated with cardiac dysfunction.

In addition, lida et al. [18] found three patterns of intrarenal venous flow, namely, the monophasic, biphasic, and continuous wave 
forms. They noted that the prognosis of the patients was the worst for the monophasic and most favorable for the continuous form [18]. Consistent with their findings, all patients in the CRS group in our study showed the biphasic venous flow pattern, not only in the renal segmental veins but also in the hepatic veins. These changes could also be associated with the cardiac dysfunction in CRS patients.

In this study, we included both age-matched and GFR-matched controls for comparison with the CKD patients with CRS in order. This group selection was made in order to assess the specificity of the newly developed ultrasound index. The fact that the positive predictability of the VVI in CRS patients was very high (96.4\%) indicates that our index is highly specific.

Our finding that the VVI correlates with diastolic dysfunction in patients with CRS indicates that diastolic heart failure might be the predominant factor leading to renal venous congestion. Even if a gold standard for the diagnosis of CRS is pending, the VVI might be integrated into a diagnostic algorithm in order to simplify the diagnosis of cardio renal disease.

Our study does have some limitations, including a small sample population and retrospective design. We intend to further validate our findings in more large-scale, multi-center trials across various ethnicities in order to justify the widespread application of this new index.

Recent studies revealed that nearly $30 \%$ of patients admitted with decompensated heart failure had an elevated creatinine concentration or a history of renal insufficiency (20). Renal dysfunction is one of the most important independent risk factors for poor outcomes and all-cause mortality in patients with heart failure (21). Thus, there is an unmet medical need for new diagnostic approaches to detect patients with CRS as early as possible.

Clinically, our findings in this study imply that it may be possible to identify almost all patients with CRS by means of a simple Doppler ultrasound examination during routine follow-up investigations in outpatient clinics.

Early detection of CRS in this manner would allow for the implementation of early interdisciplinary intervention strategies. This would, in turn, reduce the socioeconomic burden of CRS patients and improve their clinical outcome.

\section{Conflict of Interest}

The authors state that there is no conflict of interest.

\section{References}

[1] Ronco C, Haapio M, House AA, Anavekar N, Bellomo R. Cardiorenal syndrome. J Am Coll Cardiol 2008; 52 (19): 1527-1539

[2] Andrukonis K, Bell C, Bodine L, McDowell EH, Reich S, Gregory T. Cardiorenal syndrome: Understanding the connections between cardiac and renal disease. JAAPA 2014; 27 (2): 12-7. doi:10.1097/01. JAA.0000442697.65104.e2

[3] Gansevoort RT, Correa-Rotter R, Hemmelgarn BR, Jafar TH, Heerspink $\mathrm{HJ}$, Mann JF et al. Chronic kidney disease and cardiovascular risk: Epidemiology, mechanisms, and prevention. Lancet 2013; 382 (9889): 339-52. doi:10.1016/S0140-6736(13)60595-4. Epub 2013 May 31
[4] Granata A, Clementi A, Virzì GM, Brocca A, de Cal M, Scarfia VR et al. Cardiorenal syndrome type 4: From chronic kidney disease to cardiovascular impairment. Eur J Intern Med 2016; 30: 1-6. doi:10.1016/j.ejim.2016.02.019. Epub 2016 Mar 6

[5] Tsuruya K.Eriguchi M Yamada S Hirakata H Kitazono T. Cardiorenal syndrome in end-stage kidney disease. Blood Purif 2015; 40 (4): 337-43. doi:10.1159/000441583. Epub 2015 Nov 17

[6] Sperry BW, Campbell J, Yanavitski M, Kapadia S, Tang WHW, Hanna M. Peripheral venous pressure measurements in patients with acute decompensated heart failure (PVP-HF). Circ Heart Fail 2017; 10 (7): pii e004130. doi:10.1161/CIRCHEARTFAILURE.117.004130

[7] Poelzl G, Ess M, Von der Heidt A, Rudnicki M, Frick M, Ulmer H. Concomitant renal and hepatic dysfunctions in chronic heart failure: Clinical implications and prognostic significance. Eur J Intern Med 2013; 24 (2): 177-182

[8] Ronco C, Di Lullo L. Cardiorenal syndrome in western countries: Epidemiology, diagnosis and management approaches. Kidney Dis 2017; 2 (4): 151-163

[9] Meier M, Winterhoff ], Fricke L, Lehnert H, Nitschke M.. Compensatory capacity of the remnant kidney after living kidney donation: long term follow up. Transplant Proc 2017; 49 (9): 1993-1998

[10] Meier M, Fricke L, Eikenbusch K, Smith E, Kramer ], Lehnert $\mathrm{H}$ et al. The serial duplex index improves differential diagnosis of acute renal transplant dysfunction. J Ultrasound Med 2017; 26: 1607-1615

[11] Hagendorff A.. Transthoracic echocardiography in adult patients - A proposal for documenting a standardized investigation. Ultraschall Med 2008; 29: 344

[12] Lang RM, Badano LP, Mor-Avi V, Afilalo J, Armstrong A, Ernande L et al. Recommendations for cardiac chamber quantification by echocardiography in adults: An update from the American Society of Echocardiography and the European Association of Cardiovascular Imaging. Eur Heart J Cardiovasc Imaging 2015; 16: 233

[13] Ismail Y, Kasmikha Z, Green HL, McCullough PA. Cardio-renal syndrome type 1: Epidemiology, pathophysiology, and treatment. Semin Nephrol. 2012; 32 (1): 18-25

[14] Grande D, Terlizzese P, lacoviello M. Role of imaging in the evaluation of renal dysfunction in heart failure patients. World J Nephrol 2017; 6 (3): 123-131. Published online 2017 May 6. doi:10.5527/wjn.v6.i3.123

[15] Viazzi F, Leoncini G, Derchi LE, Pontremoli R. Ultrasound Doppler renal resistive index: $A$ useful tool for the management of the hypertensive patient. Journal of Hypertension 2014; 32 (1): 149-153. doi:10.1097| HJH.0b013e328365b29c

[16] Tublin ME, Bude RO, Platt JF. Review. The resistive index in renal Doppler sonography: Where do we stand? AJR Am J Roentgenol 2003; 180: 885-892

[17] Parolini C, Noce A, Staffolani E, Giarrizzo GF, Costanzi S, Splendiani G. Renal resistive index and long-term outcome in chronic nephropathies. Radiology 2009; 252: 888-896

[18] lida N, Seo Y, Sai S, Machino-Ohtsuka T, Yamamoto M, Ishizu T et al. Clinical implications of intrarenal hemodynamic evaluation by doppler ultrasonography in heart failure JACC Heart Fail. 2016; 4 (8): 674-82. doi:10.1016/j.jchf.2016.03.016. Epub 2016 May 11

[19] Nijst P, Martens P, Dupont M, Tang WHW, Mullens W. Intrarenal flow alterations during transition from euvolemia to intravascular volume expansion in heart failure patients. JACC Heart Fail 2017; 5 (9): 672-681

[20] Bock JS, Gottlieb SS. Cardiorenal Syndrom. New perspectives. Circulation 2010; 2010; 121: 2592-2600

[21] Forman DE, Butler ], Wang Y, Abraham WT, O’Connor CM, Gottlieb SS et al. Incidence, predictors at admission, and impact of worsening renal function among patients hospitalized with heart failure. J Am Coll Cardiol 2004; 43: 61-67 
Notice

This article was changed according to the erratum on 04.04.2022.

\section{Erratum}

In the abovementioned article, the name of a co-author was indicated incorrectly. Correct is: Wolfram Johannes Jabs. This was corrected in the online version on 04.04.2022. 